\title{
Hormones of the Anterior Lobe of the Pituitary Gland
}

$I^{\mathrm{T}}$ is now generally admitted that the functions of the pituitary gland (or hypophysis) are mediated by the secretion of a number of hormones from its different parts; although no active principle has yet been isolated in the pure state, the fractionation of extracts has led to the preparation of solutions having only a part of the physiological activity of the original extract. Differences of opinion exist as to the number of hormones actually present, which can only be settled when they are finally isolated as chemical individuals. Our knowledge of the functions of the posterior lobe preceded that of the anterior, but within the last few years, with improvement in both chemical and surgical technique, and also following the discovery that hormones regulating certain of the sexual activities of the body are excreted in the urine, great advances have been made also in our knowledge of the functions of the anterior lobe.

It appears probable that a number of different hormones are secreted by this lobe, but attention has been directed especially to those stimulating growth and the sexual glands. One of the pioneers in this work has been H. M. Evans, of the University of California ; the results of his researches, carried on over the last decade, are now available for study, in the form of a detailed monograph*. Although the association of overfunction of the pituitary with body overgrowth (gigantism or acromegaly) and of its underfunction with dwarfism has been frequently confirmed, it was not until 1921 that Evans and Long succeeded in preparing an extract of ox anterior lobes which stimulated growth in mammals. This was due to the facts that the growth hormone is a complex substance chemically resembling the proteins, is extraordinarily labile and can only be detected when administered frequently and parenterally to suitable animals. Adult female rats more than five months old (which have therefore ceased to increase in weight), are injected intraperitoneally daily for a period of 20 days; groups of four to six animals are used and they are weighed every five days. Gains in weight of 25-100 gm. can be obtained according to the dose given ; the relationship between the logarithm of the dose and the gain in weight was found to be approximately linear. E. Bierring and E. Nielsen (Biochem. J., 26,1015 ; 1932) have compared the composition of injected growing rats with that of normal growing rats and find that the former show a greater retention of water, but that the solid matter assimilated to the body tissues contains a much greater proportion of protein and less of ash and fat than that laid down by normal animals. About three quarters of the gain in weight of the

\footnotetext{
* The Growth and Gonad-Stimulating Hormones of the Anterior Hypophysis. By H. M. Evans, K. Meyer and M. E. Simpson, in collaboration with A. J. Szarka, R. I. Pencharz, R. E. Cornish and F. L. Reichert. Memoirs of the University of California. Vol. 11. Pp. 446. (University of California Press Berkeley, California, 1933.)
}

injected animals is due to water retention, and three quarters of the dry matter deposited consists of protein.

The method of extraction recommended by Evans and his co-workers is briefly as follows: frozen ox anterior lobes are minced and extracted with water made alkaline with baryta; the mixture is centrifuged and the solution brought to $p \mathrm{H} 8$ with sulphuric acid and again centrifuged. The solution is then acidified and poured into excess of acetone ; the precipitate is filtered off and dried. This powder is stable but still contains the gonad stimulating hormone. On extraction with 95-98 per cent acetic acid, the latter is destroyed and the growth hormone can be precipitated from solution by acetone in the presence of quinine sulphate. Trichloracetic acid precipitates the growth hormone and part of the gonad stimulating hormone from aqueous solutions of the powder; in the supernatant fluid the latter can be obtained free from the former by precipitation with flavianic acid, which is then removed by 80 per cent alcohol containing 1-2 per cent ammonia. The purer growth hormone preparations are highly active in a daily dose of $5 \mathrm{mgm}$.

Hypophysectomised rats show a greater response to the growth hormone regardless of age or length of time after removal of the gland. Experiments with a hypophysectomised puppy are also described; the pituitary gland was removed when the animal was 8 weeks old; the operation was followed by complete cessation of growth. Daily intraperitoneal injections of the growth hormone resulted in a marked increase in weight and size, so that the animal finally became larger than its litter mate control. Signs of acromegaly, however, did not develop. The ovarian follicles showed considerable development and the thyroid was hyperplastic. Similar injections into a normal female resulted in the development of partial acromegaly, some gigantism and diabetes mellitus. A male, however, only developed adiposity. In dachshunds, the injections increased the size of the animals owing to increase in size of the skull and vertebræ, but the achondroplastic form of the short extremities was not altered; a male developed diabetes. The only outstanding acromegalic feature was a folding of the skin of the head and extremities. These results lend strong support to the generally accepted view that gigantism and acromegaly in human beings are due to over-secretion by the anterior lobe of the pituitary gland.

The gonad stimulating hormone (or hormones) is responsible for the normal development and maintenance in a state of functional activity of the sex glands, with the accessory organs and secondary sex characters. In the female the ovaries themselves respond readily to the hormone; in the male the accessory organs show the most striking effects. Immature female rats were used by Evans 
and his colleagues for the assay of their preparations; injections were made on three days, the vaginal orifice examined on the fourth and fifth days, smears being taken as soon as it had opened, and the animals killed and examined about 96-100 hours after beginning treatment.

The hormone was prepared from ox anterior lobe (a poor source), from the serum of pregnant mares (a good source), and from the urine of pregnant women. The acetone powder from the alkaline extract of anterior lobes is dissolved in water and the reaction of the solution adjusted to give maximum precipitation: the greater part of the hormone remains in solution and is precipitated by flavianic acid, which can be removed afterwards by use of alcohol-ammonia mixture. Alternatively, the powder may be extracted with 50 per cent pyridine, 50-60 per cent alcohol or acetone containing 2-4 per cent ammonia; the hormone is then precipitated by increasing the alcohol or acetone to 85 per cent and adding a little acetic acid or salt. Pregnant mare serum was treated directly with acidified acetone and the powder purified by the methods used in the case of preparations from anterior lobes. From the urine of pregnant women the hormone (called prolan by Aschheim and Zondek its discoverers), was precipitated by excess of alcohol. The precipitate was extracted with dilute acid and the prolan reprecipitated with alcohol; the powder was purified by extraction with acetone-ammonia mixture. The minimum dose of the purest preparations was about $0.05 \mathrm{mgm}$.

Although preparations from these different sources all stimulate the gonads, yet they show differences in their chemical properties and biological effects. Prolan, for example, is more sensitive to both acid and alkali than preparations from pregnant mare serum; the latter, but not the former, give off hydrogen sulphide on treatment with alkali. However, the differences in chemical properties may be due to differences in the associated impurities. Differences in the biological effects produced are not so easily explained. Even though the minimal doses of different preparations may be the same, larger doses may have widely different effects on the ovary : thus increasing the dose of prolan increases the size of the ovaries at most four times, whilst with preparations from pregnant mare serum, there is a rough proportionality between dose and size up to about twenty-five times the minimal dose.

Evans and his co-workers have not been able to separate the gonad stimulating hormone into follicle stimulating and luteinising factors, corresponding to the prolans $A$ and $B$ of Aschheim and Zondek. A solution which is predominantly follicle stimulating at one dose level may produce corpora lutea at another level or when the injections are continued beyond the usual three-day period; the predominant effect may depend on the amount of purification to which the extract has been subjected. The type of response also depends in part on the time at which the examination is made after beginning the injections. There are indica- tions that the presence of corpora lutea inhibits further development of the ovary : the occurrence of ovulation depends on the size of the ovary and the dose given. Hypophysectomised female rats were less sensitive than normal animals and the response to prolan was much less than that to extracts of pregnant mare serum; simultaneous administration of the growth promoting hormone diminished the response. Substitution therapy failed to induce the rhythmic changes in the vagina characteristic of the œestrous cycle, a continuous œstrous reaction only being obtained. Pregnancy was not observed owing to failure of implantation, but it could be maintained in animals, hypophysectomised after implantation, by injection of mixtures of growth- and gonad-stimulating hormones.

In hypophysectomised female dogs, prolan had no effect on the genital system even in large doses, and when the system showed a marked degree of atrophy, an anterior lobe extract also had no effect. A mixture of the two preparations, however, stimulated the genitalia within ten days; the vulva increased to a size greater than that observed in normal œstrus in a litter mate control, the mammary gland and uterus showed marked development and the ovary was much enlarged and contained many corpora lutea. This result may be contrasted with some experiments on the hypophysectomised ferret recently published by $\mathbf{M}$. K. McPhail (Proc. Roy. Soc., B, 114, 128; 1933). Anterior lobe extract alone produced extensive theca luteinisation of small follicles, but no development of large follicles: prolan alone caused many follicles to undergo partial growth, which, however, terminated in atresia : the vulva showed partial œestrous swelling. A mixture of the two preparations produced usually only theca luteinisation.

Without referring to other work in detail, it maybe stated that several workers in addition to Aschheim and Zondek have adduced evidence that the follicle stimulating and luteinising hormones from the anterior lobe are separate entities. The synergistic action with prolan may depend on the proportions of these factors present in different preparations. Apart from the chemical difficulties of preparing the hormones in a pure state, the facts that they act in succession, or if really a single entity initiate a series of reactions, introduces a complication into the evaluation of the biological tests, which only further work with a standardised technique can clarify.

In immature male rats, doses of gonad stimulating hormone sufficient to produce enlargement of the ovaries in immature females produced little or no increase in the weight of the testes, although the accessory organs grew markedly and attained the size characteristic of these organs in young adults. Larger doses of hormone, however, increased the weight of the testes. Senile males also responded by increase in weight of the accessory organs. In hypophysectomised males injections of the hormone caused regeneration of the atrophied testes, the seminal vesicles became enlarged and filled with fluid and spermatogenesis was resumed; the 
replacement therapy was complete since normal litters were sired, and the testes appeared normal on histological examination.

The atrophy of the thyroid and adrenal glands after removal of the pituitary was not repaired by injection of gonad stimulating hormone, but extracts containing the growth hormone maintained or restored the weight of these organs, although histologically the normal structure was not completely regained. Evans's results do not show whether it is the growth hormone or some other active principles in the extracts which are responsible for these effects. The cachexia commonly observed in hypophysectomised rats was also relieved by injections of the growth hormone.

The data on which the workers in the University of California base the conclusions briefly reviewed above are available in detail in the monograph now under notice. The methods described should be of value to other investigators and their results should form the basis of further research in this important field.

\section{The British Association Tables of Bessel Functions}

$\mathrm{I}^{\mathrm{N}}$ 1888 a Committee was appointed by the British Association for the purpose "of considering the possibility of calculating tables of certain mathematical functions, and, if necessary, of taking steps to carry out the calculations, and to publish the results in an accessible form". The Committee had the late Lord Rayleigh for chairman, and 'Mr.' A. Lodge for secretary, and the other members were 'Sir W. Thomson', Cayley, Price, Glaisher, Greenhill and Hicks. Bessel functions were among the functions considered, and their calculation became the chief work of the Committee. Tables appeared in the Reports of the Association for 1889, 1893 and 1896. In 1907 the Committee reported on "The further tabulation of Bessel functions", and in 1909 stated that it was "also considering the advisability of collecting all existing tables of Bessel functions and publishing them in a form easily accessible to all students". During the next few years tables of Bessel functions of various types appeared regularly, and in 1915 it was reported that "the order of calculation is being arranged in accordance with the real urgency of the tables, and the stage is now coming in sight at which the Committee will be able, as authorised already by the Association, to publish a volume of fairly complete tables of the more important transcendental functions".

The Committee's hopeful plans, like others, were, however, hurled to emptiness, and although the Committee remained in existence and many tables were printed in its reports, it was not until after the Glasgow meeting of the Association in 1928 that the preparation of the long foreseen volume was taken seriously in hand; and when in 1931 a volume appeared, it contained no tables of Bessel functions. The reasons for this, and the problems confronting the Committee, were explained in the preface :

". . . It was apparent from the first that the simple plan of reprinting existing material would produce a volume neither useful nor creditable. There were gaps in the ranges of the arguments of some of the functions, natural when the tabulation had been performed at different times for special purposes, but intolerable if tables were to be issued for general use. In the case of the Bessel functions, the functions tabulated did not form in any sense a complete collection. Lastly, the original tables offered no facilities for interpolation. Two years ago the Committee decided that these difficulties must not impede publication indefinitely, and that, if the Bessel functions were reserved for an independent volume, definite progress could be made."

Since 1929, therefore, the work on Bessel functions has been independent both of the pre. paration of volumes of tables of other functions and of the computation from time to time of special tables in response to current demands. The aim of the Committee is to publish as complete and uniform a set of tables of Bessel functions as possible; the majority will be derived from the reports, but several will be new. It is estimated that the tables will extend to nearly five hundred pages, and it is proposed to issue two volumes, partly to avoid an unwieldy book and partly to expedite publication. The material for the first volume is in an advanced state, more than threequarters of the estimated 280 pages being ready for the printer.

Unfortunately, financial difficulties have now to be overcome. It is not to be expected that an undertaking of this sort can be a paying proposition. The mathematicians concerned have given their services, but the mere cost of production will be between $30 \mathrm{~s}$. and $£ 2$ a page. The calls on the British Association are far more miscellaneous, far more extensive, than in the spacious days when $£ 500$ could cheerfully be voted for a single object. At the moment, a sum of $£ 150$, which includes a grant of $£ 50$ from the Royal Society for this purpose, has been set aside by the Council of the Association, but this is little more than an earnest of belief in the Committee's plan. Unless further help is forthcoming, there is a serious danger that the enterprise to which a multitude of volunteers have given their leisure during nearly half a century will be completed by the enthusiastic drudgery of the Committee which has accepted it as a heritage, only to rest-a manuscript almost too precious to be consulted-in a fire-proof safe. It ought to be sufficient, by directing attention to this possibility, to ensure that funds will be provided to enable the two volumes to be published and thus to make available the results of so many years of voluntary work on behalf of mathematical students and others. 